\title{
Human intestinal epithelial cells secrete interleukin-1 receptor antagonist and interleukin- 8 but not interleukin- 1 or interleukin-6
}

R Daig, G Rogler, E Aschenbrenner, D Vogl, W Falk, V Gross, J Schölmerich, T Andus

\begin{abstract}
Background-There is growing evidence that intestinal epithelial cells (IECs) are involved in the mucosal immune system. Aim-To assess the pattern of cytokines secreted by IECs and lamina propria mononuclear cells (LPMNCs). To achieve this, the expression and secretion of interleukin (IL)-1, IL-1 receptor antagonist (IL-1ra), IL-6, and IL-8 in human primary colonic and ileal IECs and LPMNCs from the same patient were studied. Methods-IECs and LPMNCs were isolated from surgical specimens or endoscopic biopsy samples. mRNA expression was investigated by northern blot analysis. Secretion of IL-1ß, IL-6, IL-8, and IL-1 ra was measured by enzyme linked immunosorbent assay.

Results-IL-1ra mRNA levels were higher in IECs than in LPMNCs in all probands. IL-8 mRNA was only present in low amounts in the IECs from two controls. In none of the specimens were IL-1 $\beta$ and IL-6 mRNA present in IECs. Transcripts encoding IL-1 $\beta$, IL-1ra, IL-6, and IL-8 were identified in LPMNC preparations of all specimens. IECs from normal mucosa produced no detectable amounts of IL-1 $\beta$ or IL-6, whereas LPMNCs did. IECs secreted some IL-8 (65 (9) pg/10 ${ }^{5}$ cells) but significantly more was generated by LPMNCs (408 (43) pg/10 cells, p<0.0001). However, IECs secreted more IL-1ra than did LPMNCs (120 (12) v 94 (11) pg/10 cells). In acute inflammation, IEC IL-1 ra secretion was significantly increased. A correlation between secreted IL-1ra and the macroscopical degree of inflammation was found in Crohn's disease $(r=0.64$, $\mathrm{p}<0.0001, \mathrm{n}=36)$ and ulcerative colitis $(r=$ $0.76, p<0.0001, n=24)$.

Conclusions-IECs from normal mucosa express and secrete IL-1ra and low amounts of IL-8, but no IL-1 or IL-6. In inflamed mucosa the secretion of IL-1ra by IECs is slightly increased but may be not sufficient to antagonise the greatly increased production of proinflammatory cytokines by LPMNCs and the IECs themselves.

(Gut 2000;46:350-358)
\end{abstract}

Keywords: intestinal epithelial cells; lamina propria mononuclear cells; mucosa; cytokines; interleukin; immune system
The classical functions of the intestinal epithelium are absorption and secretion of fluids, electrolytes, and nutrients. Furthermore, the intestinal epithelial cells (IECs) form the primary physiological barrier against potentially pathogenic microorganisms and a multitude of antigens in the gut lumen. Damage of the epithelial barrier is followed by bacterial invasion and inflammation of the mucosa.

In addition, during the last few years more and more data have accumulated showing that IECs play an active role in the intestinal immune system. They express HLA-DR after stimulation with interferon- $\gamma \cdot{ }^{1-5}$ After stimulation with interferon or interleukin (IL)-1, they show enhanced expression of adhesion molecules - for example, intercellular adhesion molecule- $1^{6}$ and lymphocyte function associated antigen (CD58). ${ }^{7}$ Intercellular adhesion molecule- 1 could be enhanced in HT-29 and Caco- 2 cells by incubation with inflammatory cytokines; however, the responsiveness to interferon- $\alpha$, tumour necrosis factor (TNF)- $\alpha$, or IL-1 treatment was different in the two cell lines. ${ }^{8}$ Furthermore, evidence was found that epithelial cells are able to respond to damage or bacterial invasion by secreting cytokines. ${ }^{9-18}$ The cytokines reported to be secreted by IECs are important inflammatory mediators such as IL-1, IL-6, and IL-8. ${ }^{19-21}$ To characterise further the participation in the mucosal immune system and the immunomodulatory function of the intestinal epithelium, in vitro studies were performed. Cytokine production has been studied in human and rat epithelial cell lines such as Caco-2, HT-29, and IEC-6. ${ }^{182-24}$ T84, HT-29, and SW620 cell lines secreted substantial amounts of IL-8 after stimulation with IL- $1 \beta$, TNF- $\alpha$ or interferon- $\gamma$, but Caco- 2 cells responded only to IL- $1 \beta$. In addition to theses cytokines, bacterial lipopolysaccharide was efficient in stimulating IL-8 release in SW620 and HT29 cells, whereas T84 and Caco-2 cells were almost unresponsive to it. ${ }^{23}$ We showed that the synthesis of IL-8 in HT-29 cells was stimulated by IL- $1 \beta$ or TNF- $\alpha .^{22}$ These data indicate that the use of different IEC lines may lead to different results

Abbreviations used in this paper: IEC, intestinal epithelial cell; LPMNC, lamina propria mononuclear cell; IL, interleukin; IL-1 ra, IL-1 receptor antagonist; ELISA, enzyme linked immunosorbent assay; TNF, tumour necrosis factor; IBD, inflammatory bowel disease; PBS, phosphate buffered saline. 
and different interpretations of their immunological function.

Several studies with epithelial cell lines have shown IL-6 secretion by IECs. Experiments with the rat-derived IEC- 6 cell line suggested that IECs could represent an important source of IL-6 in inflammatory responses at the intestinal mucosa and that transforming growth factor- $\beta$ could potentiate this function. ${ }^{24} \mathrm{~A}$ recent study showed IL-1 induced and NF- $\mathrm{NB}$ mediated IL-6 production in Caco-2 cells. ${ }^{25}$ Salmonella typhi has been shown to stimulate IL-6 secretion in the small intestine epithelial cell line Int407. ${ }^{26}$ However, in a study using in situ hybridisation of human mucosa, IL-6 mRNA was not found in the epithelial cells except in one patient. ${ }^{27}$ In addition, in murine intestine, IL-6 mRNA was not located in the epithelial cell layer. ${ }^{28}$

Recently we and others showed an imbalance of the IL- 1 system in colonic mucosa during inflammation. ${ }^{29}{ }^{30}$ IL- $1 \alpha$ and IL- $1 \beta$ were significantly increased in the inflamed mucosa from patients with inflammatory bowel disease (IBD) and also in that from non-IBD patients compared with healthy controls. ${ }^{29}$ The ratio of IL-1 $(\alpha+\beta)$ to IL-1 receptor antagonist (IL-ra) was significantly decreased, leading to a change in the balance of proinflammatory and antiinflammatory cytokines. IL-8 mRNA detected by in situ hybridisation was also increased in inflamed colonic mucosa. ${ }^{31}$ The signal for IL-8 could only be found in the lamina propria, indicating intestinal macrophages as the major source of IL-8. Therefore we concluded that IL-8 is mainly produced in inflammatory cells in the lamina propria of the colon in IBD and correlates with mucosal inflammation. ${ }^{31}$ These results were in contrast with our findings in HT-29 cells, which responded to stimulation with IL- 8 secretion. ${ }^{22}$

As cell lines dedifferentiate in culture, we developed a culture system for primary cultures of human IECs. ${ }^{32}$ Using this new system, we were able for the first time to compare the expression and secretion of cytokines by IECs and the corresponding lamina propria mononuclear cells (LPMNCs) from the same patient.

\section{Materials and methods} PATIENTS

Colonic tissue was obtained from 12 patients undergoing surgical resection for colorectal carcinoma. The tissue was taken at least $5 \mathrm{~cm}$ distant from the tumour. In addition, surgical specimens from three patients with Crohn's disease and two with ulcerative colitis were obtained. Colonic mucosal biopsy specimens (six per patient) were taken from 63 patients and ileal biopsy specimens from 20 patients during colonoscopy for different reasons.

The mucosa from the control patients was macroscopically normal. None of the patients had received corticosteroids, mesalamine, sulphasalazine, or immunosuppressive drugs that would affect cytokine secretion.

The degree of inflammation of the specimens was graded endoscopically ${ }^{31}:+=$ low degree of inflammation (increased granularity and friability of the mucosa in ulcerative colitis; single small aphthous lesions in Crohn's disease) $;++=$ moderate inflammation (mucous membranes, spontaneous bleeding, and small ulcers in ulcerative colitis; multiple aphthous lesions, and small ulcers in Crohn's disease) $+++=$ severe inflammation (large ulcers in ulcerative colitis; large ulcerous lesions in Crohn's disease).

Colitis was regarded as non-specific when histological examination showed inflammatory infiltrates in the mucosa that were not typical of IBD, and treatment with antibiotics led to complete restitution. Stool cultures from these patients did not show any specific pathogen and were negative for Clostridium difficile toxin.

The study was approved by the ethics committee of the University of Regensburg and performed in accordance with the declaration of Helsinki.

ISOLATION OF COLONIC AND ILEAL EPITHELIAL CELLS

A method for the isolation of viable epithelial cell preparation has been developed based on standard protocols and is described in more detail elsewhere. ${ }^{32}$ In brief, mucosa was stripped from submucosa within 30 minutes of bowel resection and rinsed several times with phosphate buffered saline (PBS). The mucus was removed by treatment with $1 \mathrm{mM}$ dithiothreitol (Serva, Heidelberg, Germany) for 15 minutes. After being washed with PBS, the mucosa was placed in $1.5 \mathrm{mM}$ EDTA in Hanks balanced salt solution without calcium and magnesium and tumbled for 10 minutes at $37^{\circ} \mathrm{C}$. This supernatant containing debris and mainly villus cells was discarded. The mucosa was incubated again with EDTA for 10 minutes at $37^{\circ} \mathrm{C}$. The supernatant was collected into a $15 \mathrm{ml}$ tube. Then the remaining mucosa was vortex-mixed in PBS and this supernatant was also collected. It contained complete crypts, some single cells, and a small amount of debris. To separate IECs (crypts) from contaminating non-epithelial cells, the suspension was allowed to sediment for 15 minutes. The cells (mainly complete crypts) were collected and washed twice with PBS. The procedure to isolate IECs from mucosal biopsy specimens was identical except that the dithiothreitol treatment was omitted. The number and viability of the cells were determined by $0.1 \%$ trypan blue exclusion. The purity of the epithelial cell preparation was checked by FACS analysis with the EP4 antibody (Dako, Hamburg, Germany), showing more than $90 \%$ of EP4 positive cells. The amount of macrophages (CD33 positive) or lymphocytes (CD3 or CD19 positive) was less than $4 \%$ each. Colonic epithelial cell cultures from surgical specimens were used for extraction of RNA, which required larger cell numbers.

CULTURE OF COLONIC AND ILEAL EPITHELIAL CELLS

The cells $\left((1-5) \times 10^{5}\right)$ were resuspended in $400 \mu \mathrm{l}$ minimal essential medium supplemented with Earle's salts, $20 \%$ fetal calf serum, 
ITS $(5 \mu \mathrm{g} / \mathrm{ml}$ insulin, $5 \mu \mathrm{g} / \mathrm{ml}$ transferrin, 5 $\mathrm{ng} / \mathrm{ml}$ selenious acid; Becton Dickinson, Heidelberg, Germany), $2 \mathrm{mM}$ glutamine, 100 $\mathrm{U} / \mathrm{ml}$ penicillin, $100 \mu \mathrm{g} / \mathrm{ml}$ streptomycin, 100 $\mu \mathrm{g} / \mathrm{ml}$ gentamicin, and $2.5 \mu \mathrm{g} / \mathrm{ml}$ fungisone (JRH Bioscience, Lenexa, Kansas, USA). Medium with stabilised glutamine was purchased from Biochrom (Berlin, Germany). Other supplements were obtained from Sigma (Deisenhofen, Germany). The IECs were seeded into collagen A (Biochrom) coated Millicell-CM cell culture plate inserts suitable for 24 well culture plates with a translucent and permeable membrane at the bottom (Millipore, Eschborn, Germany). The cells were incubated at $37^{\circ} \mathrm{C}$ in air with $10 \% \mathrm{CO}_{2}{ }^{32}$

ISOLATION OF LPMNCs

After removal of IECs, the mucosa was digested enzymically by incubation with 1 $\mathrm{mg} / \mathrm{ml}$ collagenase (collagenase type I; Sigma), $0.3 \mathrm{mg} / \mathrm{ml} \mathrm{DNAse}$ I (Boehringer Mannheim, Mannheim, Germany), and $2 \mathrm{mg} / \mathrm{ml}$ hyaluronidase (Sigma) at $37^{\circ} \mathrm{C}$ for 30 minutes. The digest was filtered and then centrifuged in Ficoll-Paque (Pharmacia, Freiburg, Germany) for 20 minutes at $700 \mathrm{~g}$. LPMNCs showed a viability of $>90 \%$ as assessed by $0.1 \%$ trypan blue exclusion.

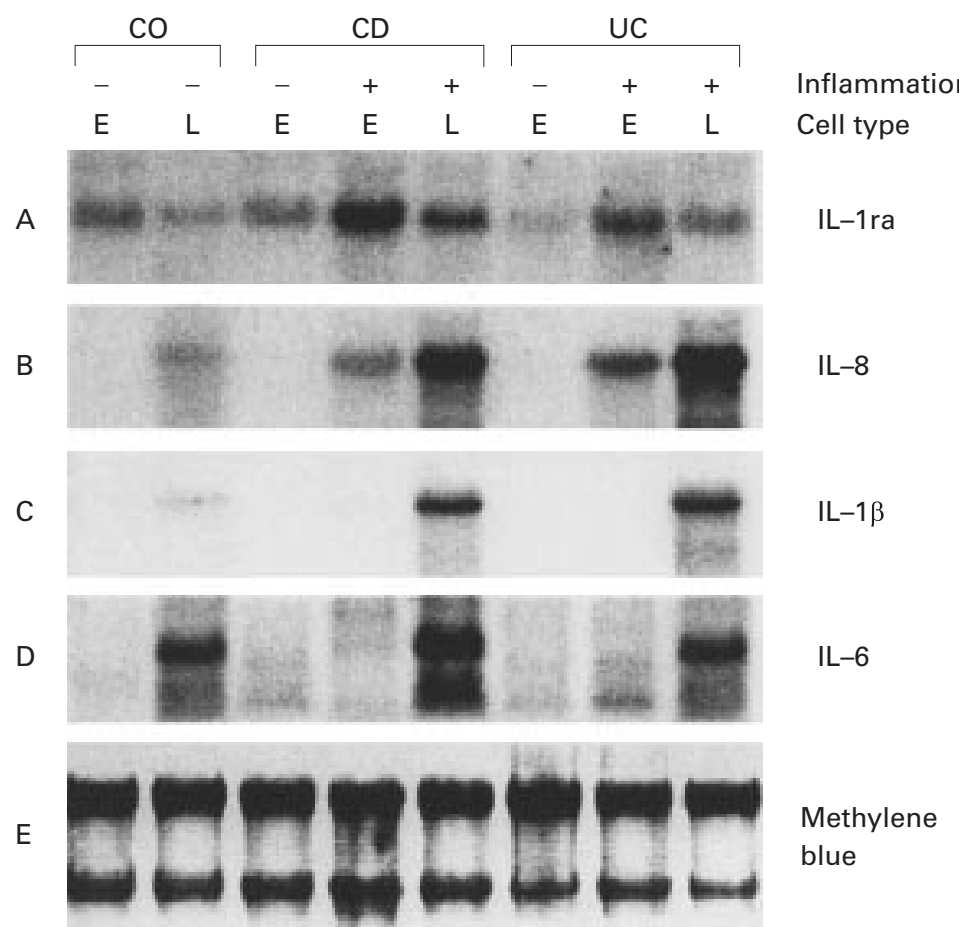

Figure 1 Northern blot analysis of interleukin (IL)-1 receptor antagonist (ra), IL-8, $I L-1 \beta$, and IL-6 mRNA. RNA was extracted from intestinal epithelial cell (IEC) or lamina propria mononuclear cell (LPMNC) preparations from five control patients (CO), three patients with Crohn's disease (CD), and two patients with ulcerative colitis (UC). Total RNA (25 $\mu \mathrm{g}$ ) extracted from IECs or LPMNCs was separated in an agarose gel containing $1 \%$ formaldehyde, transferred to nylon membranes, and hybridised with $\left.{ }^{32} \mathrm{P}\right] d C T P$ labelled cDNA fragments from IL-1ra cDNA (A), IL-8 cDNA (B), IL $1 \beta$ (C), and IL-6 cDNA (D). Methylene blue staining was performed to confirm equal loading of RNA (E). E, Epithelial cells; L, lamina propria mononuclear cells. Expression of IL-1 ra $m R N A$ was higher in IECs than LPMNCs and increased during inflammation. $I L-8 m R N A$ was only expressed in two of five IEC preparations isolated from normal mucosa and was increased in mucosal inflammation but always to a lesser extent than in LPMNCs. IL-1 $\beta$ and IL-6 mRNA could not be found in the IEC preparation but were always present in LPMNCs.
NORTHERN BLOT ANALYSIS

IECs and LPMNCs freshly isolated from surgical specimens were homogenised with a sonifier (Bandelin) at 50\% of maximal power for five seconds. Isolation of total RNA was based on guanidinium isothiocyanate according to standard procedures. RNA $(25 \mu \mathrm{g})$ was separated in a formaldehyde-containing agarose gel, transferred to a nylon membrane (Appligene, Illkirch, France), and cross linked by exposure to ultraviolet light using a Stratalinker (Stratagene, Heidelberg, Germany). The transfer was controlled by staining the membrane with methylene blue. Hybridisation was carried out with cDNA probes labelled with $\left[{ }^{32} \mathrm{P}\right] \mathrm{dCTP}$ by random priming (Stratagene) (190 bp fragment of IL-1, $455 \mathrm{bp}$ fragment of IL-1ra, $587 \mathrm{bp}$ fragment of IL-6, and $244 \mathrm{bp}$ fragment of IL-8). Agarose gel electrophoresis, RNA transfer, and hybridisation of the membrane were performed as described previously. ${ }^{22}$ The membranes were exposed to $x$ ray films with intensifying screens (Hyperfilm MP; Amersham, Braunschweig, Germany) for $1-2$ days at $-70^{\circ} \mathrm{C}$.

DETERMINATION OF IL-1, IL-1ra, IL-6, AND IL-8 PROTEIN

Supernatants of IEC and LPMNC cultures were collected after 24 hours. Cytokines were measured by enzyme linked immunosorbent assay (ELISA; Biotrak-Amersham, Braunschweig, Germany) according to the manufacturer's protocol. The results were expressed as pg cytokine $/ 10^{5}$ cells.

WESTERN BLOTTING FOR IL-1ra

Peripheral blood monocytes and IECs cultured for 24 hours with or without $10 \mathrm{ng} / \mathrm{ml} \mathrm{TNF}$ were lysed in a buffer containing $4 \mathrm{mM}$ Hepes, $320 \mathrm{mM}$ sucrose, $1 \mathrm{mM}$ EDTA, $0.1 \mathrm{mM}$ dithiothreitol, $1 \mathrm{mM}$ phenylmethanesulphonyl fluoride, $1.5 \mathrm{mM}$ pepstatin, $2 \mathrm{mM}$ leupeptin, $0.7 \mathrm{mM}$ aprotinin, and $0.1 \%$ CHAPS on ice for 20 minutes. After sonification for five seconds, protein extracts were centrifuged for 10 minutes at $15000 \mathrm{U} / \mathrm{min}$. Protein concentration in the supernatant was determined using the bicinchoninic acid (BCA) test from Bio-Rad. Medium proteins were precipitated by incubation with ice cold acetone $(8: 1, \mathrm{v} / \mathrm{v})$ for one hour on ice. Centrifugation was performed for 30 minutes at $6000 \mathrm{~g}$ in a Heraeus Megafuge $1.0 \mathrm{R}$ at $4^{\circ} \mathrm{C}$. Proteins were resuspended in reducing sample buffer. Then $80 \mu \mathrm{g}$ of total protein from each sample was loaded on a sodium dodecyl sulphate/15\% polyacrylamide gel. Recombinant human IL1 ra (R\&D Systems, Wiesbaden, Germany) was used as a positive control. After electrophoresis, protein was transferred to a nitrocellulose membrane. Blots were blocked with 5\% non-fat milk in PBS and probed with 1:3000 dilutions of IL-1 ra monoclonal antibody (R\&D Systems) in 5\% non-fat milk/PBS. Immunoblots were then processed with 1:5000 diluted horseradish peroxidase conjugated anti-goat IgG (Dianova, Hamburg, Germany) using the enhanced chemiluminescence plus (ECL Plus) western blotting detection system kit (Amer- 
sham Pharmacia Biotech). The blots were exposed to Hyperfilm (ECL, Amersham) at room temperature.

\section{STATISTICAL ANALYSIS}

Data are expressed as mean (SEM). The statistical analysis was performed by the Kruskal-Wallis $\mathrm{H}$ test or the Mann-Whitney rank sum test for non-parametric data. Differences with a $p$ value of $<0.05$ were considered significant.

\section{Results}

EXPRESSION OF CYTOKINE MRNA

Total RNA extracted from freshly isolated IECs and LPMNCs from five colonic surgical specimens without inflammation (carcinoma patients), two surgical specimens from patients with ulcerative colitis, and three specimens from patients with Crohn's disease was subjected to northern blot analysis to screen for IL-1 $\beta$, IL-1ra, IL-6, and IL-8 expression.
As the epithelial cell preparations contained some $(<10 \%)$ non-epithelial cells, RNA from LPMNCs was isolated from the same surgical specimens for comparison.

IL-1ra

Low expression of IL-1ra was found in the IECs from all control patients and also in the uninflamed mucosa of patients with Crohn's disease (fig 1, lane A). It was higher in inflamed regions of patients with IBD. In the IECs of all patients, IL-1ra mRNA levels were as high as, or higher than, in LPMNCs (fig 1, lane A). The integrity of the extracted RNA and gel loading was confirmed by methylene blue staining of the nylon membrane (fig 1, lane E).

\section{IL-8}

IL-8 mRNA was only present in low amounts in IECs from two of the five control patients. IL-8 expression was also low in uninflamed areas of the specimens from patients with
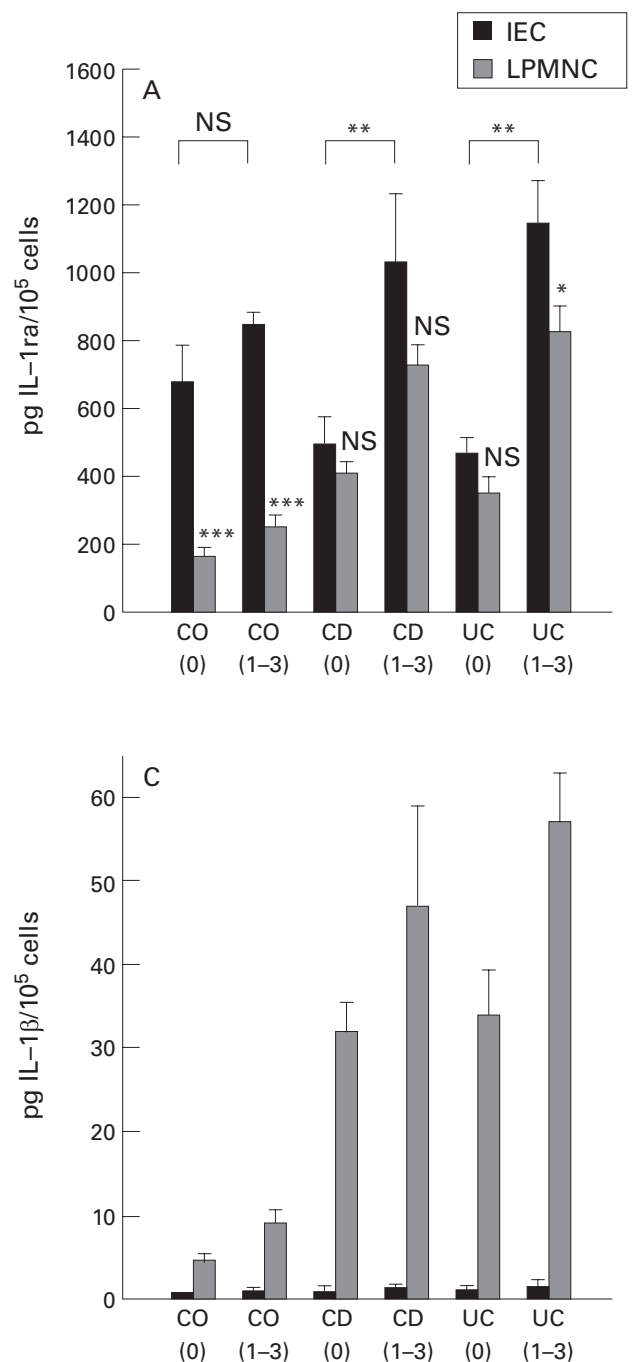
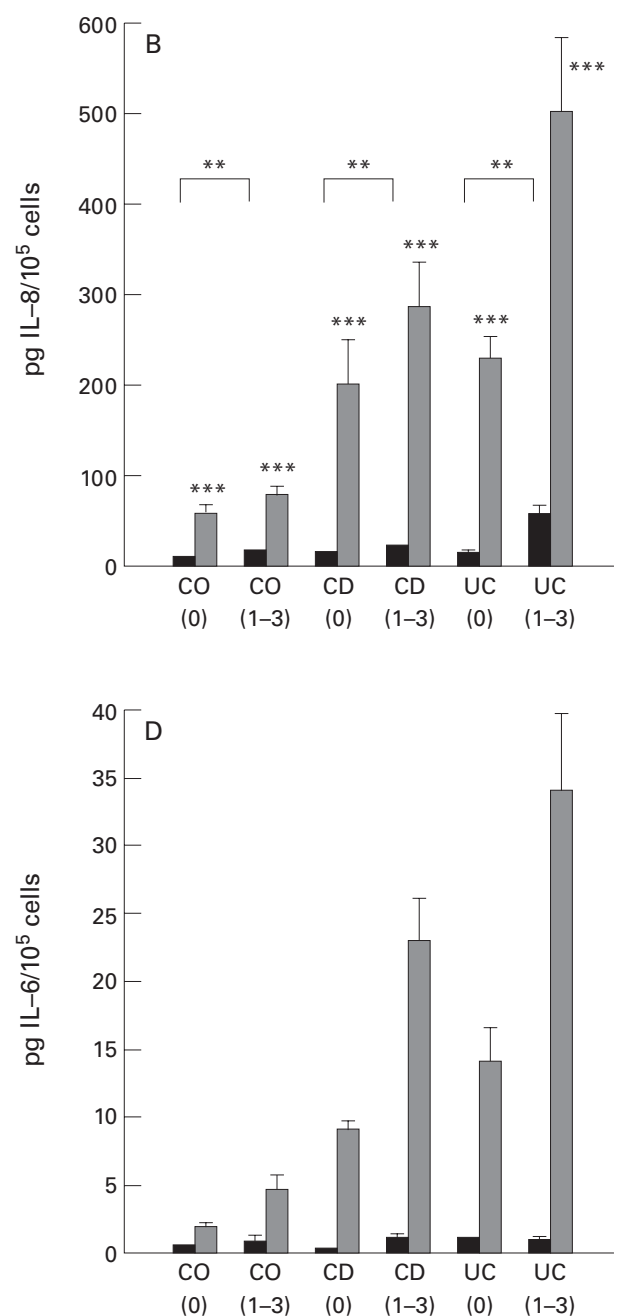

Figure 2 Cytokine content of freshly isolated intestinal epithelial cells (IECs) and lamina propria mononuclear cells (LPMNCs) from normal and inflamed mucosa. Interleukin (IL)-1 receptor antagonist (ra) ( $A$ ), $I L-8(B), I L-1 \beta$ (C), and IL-6 (D) protein was determined from freshly isolated and homogenised IECs from 50 biopsy or surgical specimens of normal mucosa, from five patients with unspecific colonic inflammation (CO), 24 patients with Crohn's disease (CD), and 14 patients with ulcerative colitis (UC) by ELISA. In addition, cytokines were determined in LPMNC homogenates from 17 control patients, five patients with unspecific colitis, nine patients with Crohn's disease, and nine patients with ulcerative colitis. Results are mean (SEM) expressed as pg cytokine $10^{5}$ cells. $0=$ not inflamed, $1=$ slightly inflamed, $2=$ moderately inflamed, $3=$ severely inflamed. ${ }^{\star} p<0.05,{ }^{\star \star *} p<0.001 v$ IECs; ${ }^{\star \star} p<0.01$ inflamed $v$ non-inflamed. 
Table 1 Interleukin (IL) 1 receptor antagonist (ra), IL-8, IL-1 $\beta$, and IL-6 content of freshly isolated intestinal epithelial cells (IECs) and lamina propria mononuclear cells (LPMNCs) normalised to cell protein

\begin{tabular}{|c|c|c|c|c|c|c|c|c|}
\hline & \multicolumn{2}{|l|}{$I L-1 r a$} & \multicolumn{2}{|l|}{$I L-8$} & \multicolumn{2}{|l|}{$I L-1 \beta$} & \multicolumn{2}{|l|}{$I L-6$} \\
\hline & $I E C$ & $L P M N C$ & IEC & $L P M N C$ & $I E C$ & $L P M N C$ & IEC & $L P M N C$ \\
\hline $\mathrm{CO}(0)$ & 7397 (1039) & 3199 (375) & $137(19)$ & $1144(145)$ & $1.8(0.6)$ & $97(14)$ & $1.4(0.2)$ & $42(5.6)$ \\
\hline $\mathrm{CO}(1-3)$ & $8973(1570)$ & $4325(380)$ & $187(17)$ & $1836(133)$ & $9.8(2.1)$ & $263(46)$ & $2.9(1.3)$ & $100(18)$ \\
\hline $\mathrm{CD}(0)$ & $5706(1056)$ & $3836(154)$ & $122(8.7)$ & $1853(436)$ & $9.9(2.9)$ & $305(45)$ & $3.1(1.0)$ & $86(1.9)$ \\
\hline $\mathrm{CD}(1-3)$ & 9301 (2188) & 5440 (748) & $216(29)$ & 2005 (194) & $30 \quad(4.4)$ & $348(80)$ & $8.8(1.6)$ & $185(34)$ \\
\hline $\mathrm{UC}(0)$ & $5914(1777)$ & $4253(533)$ & $181(69)$ & $2283(197)$ & $(5.9)$ & 339 (39) & $13 \quad(1.6)$ & $99(12)$ \\
\hline UC (1-3) & $17856(2013)$ & $10216(348)$ & $1162(206)$ & $6624(1812)$ & (6.8) & $697(24)$ & $17 \quad(2.2)$ & $422(32)$ \\
\hline
\end{tabular}

Cytokines were measured in homogenised, freshly isolated IECs from 50 control patients (CO, 0), five patients with unspecific intestinal inflammation (CO, 1-3), 24 patients with Crohn's disease (CD), and 14 patients with ulcerative colitis (UC). Cytokines were also measured in freshly isolated LPMNCs from 17 control patients, five patients with unspecific intestinal inflammation, nine patients with Crohn's disease, and nine patients with ulcerative colitis.

Data (mean (SEM) are expressed as pg cytokine/mg total protein.

0 , mucosa not inflamed; 1 , slightly inflamed; 2 , moderately inflamed; 3 , severely inflamed.

Crohn's disease. It was higher in IECs from inflamed mucosa from patients with ulcerative colitis and Crohn's disease (fig 1, lane B). In LPMNCs from control patients, IL-8 expression was low, but higher than in IECs. In patients with IBD, there was an obvious increase in IL-8 expression by LPMNCs. In all patients investigated, IL-8 expression was higher in LPMNCs than in IECs (fig 1, lane B).
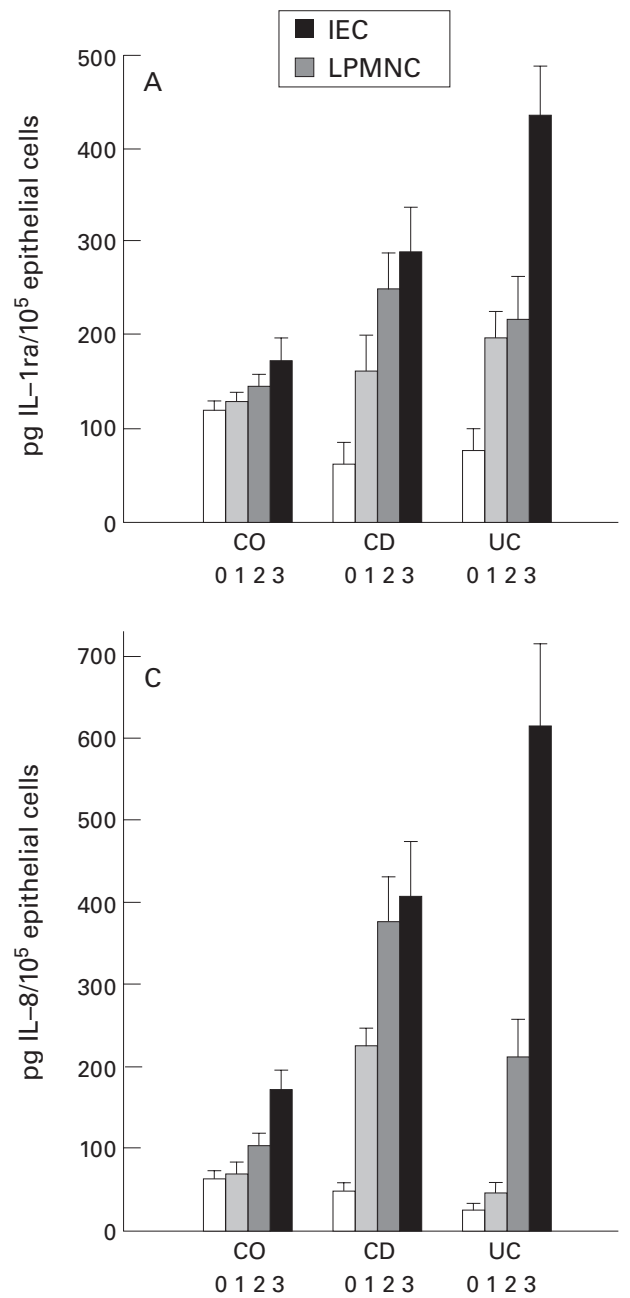

$I L-1 \beta$ and $I L-6$

In contrast with IL-1 ra and IL- 8 mRNA, in none of the specimens was IL- $1 \beta$ (fig 1 , lane C) and IL-6 (fig 1, lane D) mRNA present in IECs. IL- $1 \beta$ expression in LPMNCs from control patients was low but was obviously increased in patients with IBD (fig 1, lane C). Already in the control specimens, IL-6 was expressed in the LPMNC fraction. This
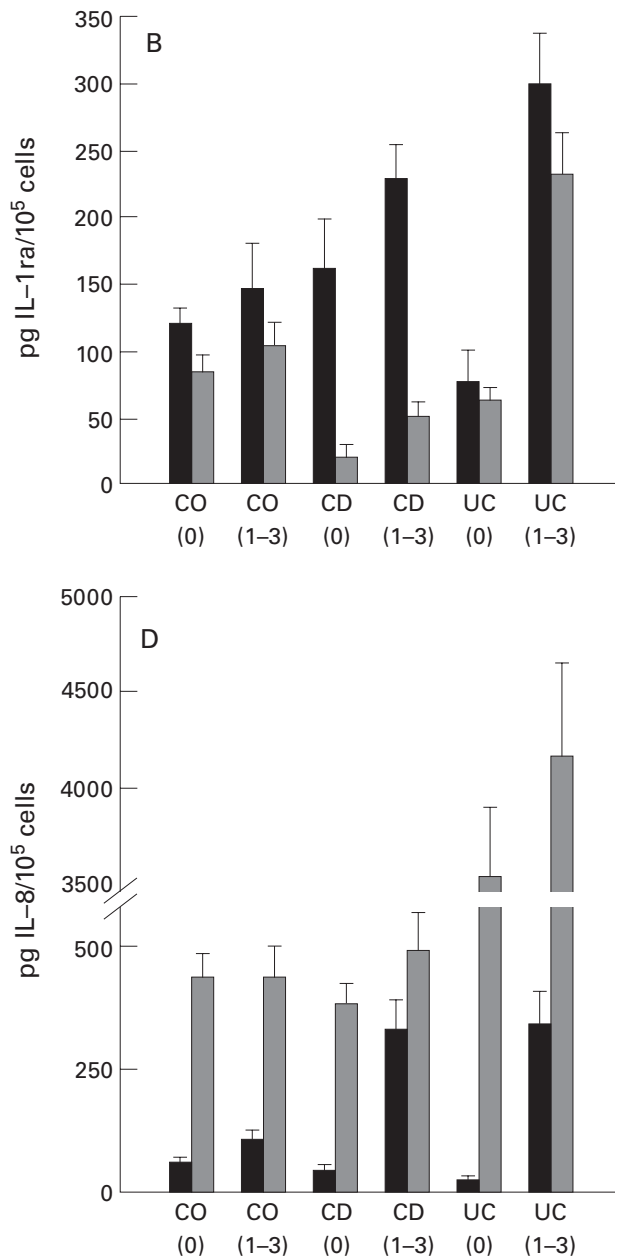

Figure 3 Secretion of interleukin (IL)-1 receptor antagonist (ra) and IL-8 by primary cultures of intestinal epithelial cells (IECs) after 24 hours. (A) IL-1ra was determined in culture medium after a 24 hour culture of primary human IECs from 72 biopsy or surgical specimens from control mucosa $(C O(0)), 12$ patients with unspecific colonic inflammation (CO (1-3)), 44 patients with Crohn's disease (CD), and 25 patients with ulcerative colitis (UC). The degree of inflammation was estimated endoscopically as described in Materials and methods: $0=$ normal mucosa, $1=$ low degree of inflammation, 2 = moderate inflammation, 3 = severe inflammation. (B) Comparison of IL-1ra secretion from IECs and LPMNCs after 24 hours of culture. In addition to IECs, LPMNCs were isolated from 29 control patients, five patients with unspecific colitis, 15 patients with Crohn's disease, and 12 patients with ulcerative colitis. $(C, D)$ IL-8 secretion from 24 hour cultured IECs and LPMNCs. For details see $(A)$ and $(B)$. Results are mean (SEM) expressed as pg/10 cells. 
expression was obviously increased in Crohn's disease, but not in ulcerative colitis.

CYTOKINE CONTENT OF FRESHLY ISOLATED IECS AND LPMNCs

To exclude post-transcriptional differences in processing of the mRNAs leading to a misinterpretation of the northern blot findings, we determined the cytokine content of freshly isolated colonic IECs and LPMNCs.

IL-1ra

In freshly isolated IECs from non-inflamed mucosa, IL-1 ra concentrations were higher in control patients than in those with Crohn's disease and ulcerative colitis, but these differences did not reach significance (fig 2A). IL-1 ra levels in IECs from control patients increased slightly when inflammation occurred (not significant compared with non-inflamed controls). However, the increase caused by inflammation was significantly higher in IECs from patients with IBD ( $p=0.04$ for Crohn's disease and $p=0.01$ for ulcerative colitis versus non-inflamed IECs) (fig 2A).

IL-1 ra levels in freshly isolated LPMNCs from control patients with and without inflammation were significantly lower than in the corresponding IECs $(p=0.0002$ and $p=0.003$ respectively) (fig $2 \mathrm{~A}$ ). In addition, freshly isolated LPMNCs from non-inflamed mucosa from patients with Crohn's disease or ulcerative colitis contained less IL-1ra than the corresponding IECs. During acute inflammation, IL-1 ra increased significantly $(\mathrm{p}=0.01)$ in the LPMNCs in Crohn's disease and ulcerative colitis but did not reach the levels of the corresponding IECs (fig 2A).

IL-8

Freshly isolated IECs from non-inflamed areas of specimens from patients with IBD and control patients contained comparable amounts of IL-8. The content of IL-8 in freshly isolated IECs from controls with colonic inflammation or Crohn's disease was still low but significantly increased compared with controls without inflammation $(\mathrm{p}=0.02$ and $\mathrm{p}=0.04$ respectively). In inflamed areas of patients with ulcerative colitis, IL-8 levels were also significantly increased ( $\mathrm{p}=0.016$ ) (fig $2 \mathrm{~B}$ ).

IL-8 protein concentration was significantly higher in LPMNCs than in the corresponding IECs in all patient groups $(p<0.001)$. It was also increased in LPMNCs in control patients during inflammation (fig 2B). In LPMNCs from non-inflamed areas of Crohn's disease mucosa, IL-8 levels were already significantly increased to 200 (51) $\mathrm{pg} / 10^{5}$ cells and in ulcerative colitis to 228 (25) pg/10 cells. During macroscopical inflammation, there was an increase in Crohn's disease to 285 (50) $\mathrm{pg} / 10^{5}$ cells and in ulcerative colitis to 501 (83) $\mathrm{pg} / 10^{5}$ cells (fig $2 \mathrm{~B}$ ).

$I L-1 \beta$ and $I L-6$

In freshly isolated IECs from all patient groups, IL-1 $\beta$ protein was at the detection limit of the ELISA $(0.5 \mathrm{pg})$. Freshly isolated LPMNCs from controls without inflammation contained
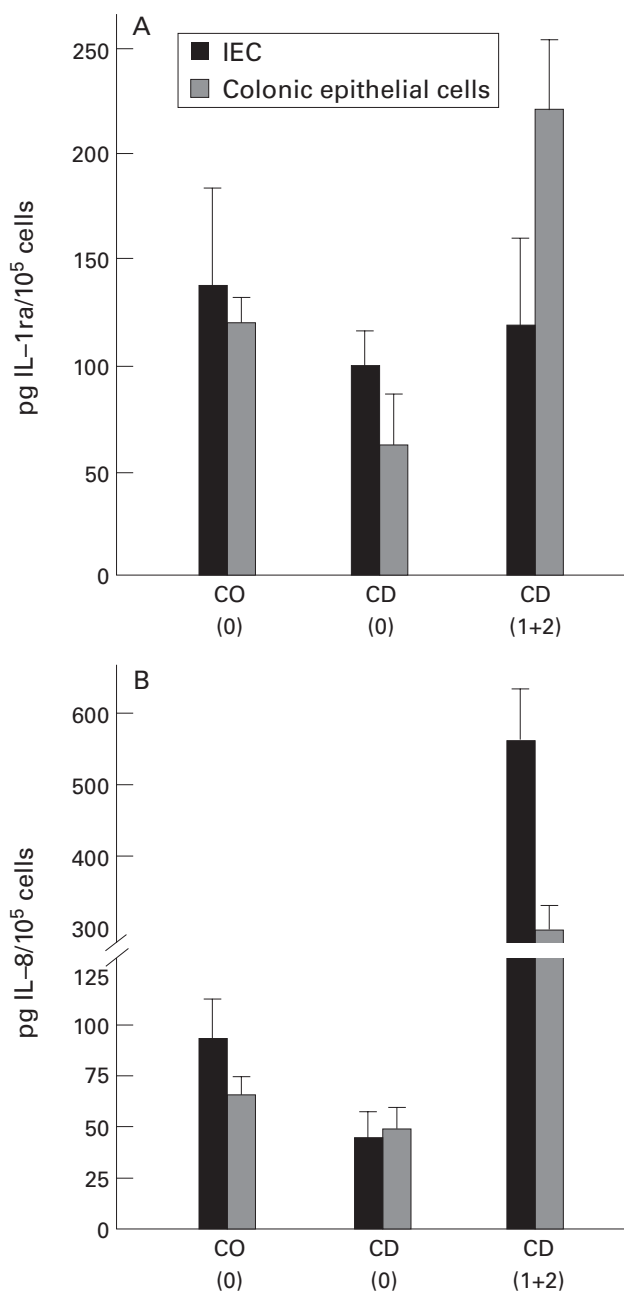

Figure 4 Comparison of interleukin (IL)-1 receptor antagonist (ra) and IL-8 secretion in isolated ileal (IEC) and colonic epithelial cells. Cytokines were determined in culture medium after 24 hours of culture. $n=7$ for controls (CO), non-inflamed Crohn's disease (CD), and inflamed CD. Data are mean (SEM) expressed as pg cytokine/10 cells. Differences between ileal and colonic epithelial cells did not reach significance.

$4.6(0.9)$ pg IL- $1 \beta / 10^{5}$ cells. In macroscopically uninvolved mucosa of patients with Crohn's disease $(\mathrm{p}=0.008)$ and ulcerative colitis $(\mathrm{p}=$ $0.04)$, IL-1 $\beta$ levels were significantly higher than in controls (fig 2C). During inflammation, IL- $1 \beta$ was increased in LPMNCs from control patients $(p=0.005)$, patients with Crohn's disease (not significant), and patients with ulcerative colitis ( $p=0.04$ ) (fig $2 \mathrm{C}$ ).

As found for IL-1 $\beta$, IL-6 levels in freshly isolated IECs were at the detection limit of the ELISA in all patient groups (0.5 pg IL-6) (fig 2D). A low amount of IL-6 was found in LPMNCs from controls, and higher levels were found in non-inflamed mucosa from patients with Crohn's disease or ulcerative colitis (fig 2D). During inflammation, IL-6 levels in LPMNCs were significantly increased in control patients $(\mathrm{p}=0.001)$, patients with Crohn's disease $(p=0.01)$, and patients with ulcerative colitis ( $p=0.03)$ (fig $2 \mathrm{D})$.

Comparable results were obtained when cytokine levels were normalised to cellular protein content (table 1). 
CYTOKINE SECRETION IN PRIMARY CULTURES OF IECs AND LPMNCs

To investigate whether the cytokine levels in freshly isolated cells corresponded to secreted and thereby bioactive cytokines, we cultured IECs and LPMNCs for 24 hours and measured cytokine levels in the culture medium.

IL-1ra

After 24 hours of culture, IECs from normal mucosa secreted 120 (12) pg IL- $1 \mathrm{ra} / 10^{5}$ cells (fig 3A). When there was acute inflammation in patients without IBD - for example, patients with diverticulitis-secretion increased, but this effect was not significant $(p=0.56)$. IECs from non-inflamed mucosa from patients with IBD showed lower secretion of IL-1 ra than controls (Crohn's disease, $62(24) \mathrm{pg} / 10^{5}$ cells; ulcerative colitis, 77 (24) $\mathrm{pg} / 10^{5}$ cells). When IL-1 ra secretion by IECs from macroscopically inflamed mucosa was analysed, there was a significantly higher secretion than from cells from an area with a low degree of inflammation (Crohn's disease, $\mathrm{p}=0.0013$; ulcerative colitis, $p=0.0002$ ) (fig 3A). There was a correlation between the secreted amounts of IL-1 ra and the macroscopical degree of inflammation (Crohn's disease, $r=0.64, \mathrm{p}<0.001$; ulcerative colitis, $r=0.76, \mathrm{p}<0.0001)$. In all groups, IECs secreted more IL-1 ra than did LPMNCs after 24 hours of culture (fig 3B).

\section{$I L-8$}

The secretion of IL-8 in supernatants of IECs cultured for 24 hours was low (fig 3C). IECs from unspecific inflamed mucosa showed a significant increase $(p=0.008$ ) (fig $3 C$ ). There was also low IL-8 secretion by IECs from noninflamed regions of IBD mucosa, which was not significantly different from normal IECs (fig 3C). IECs from patients with macroscopically severely inflamed mucosa secreted significantly more IL-8 than those from non-

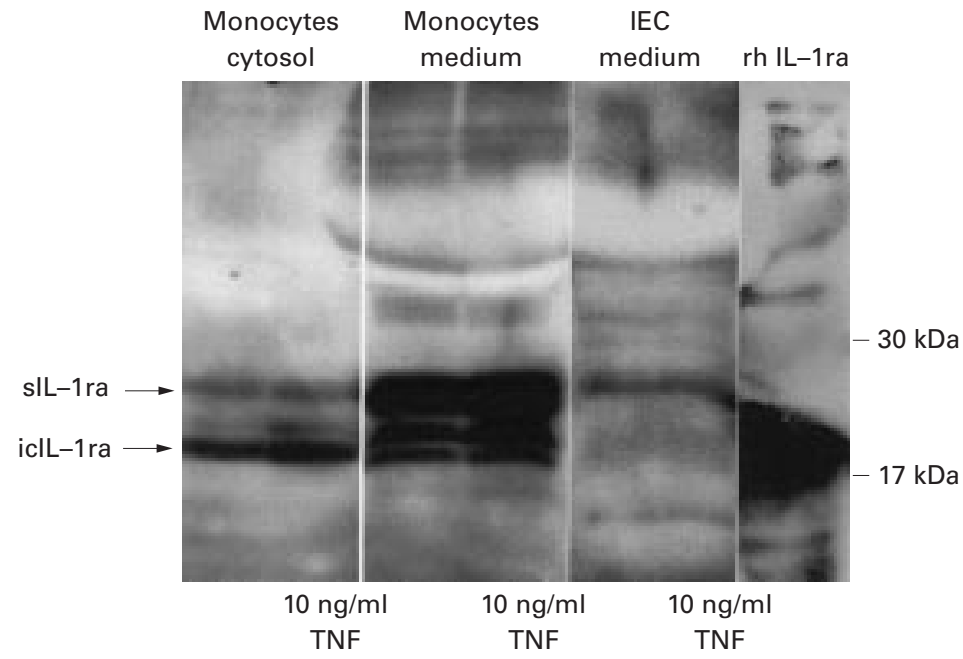

Figure 5 Identification of interleukin (IL)-1 receptor antagonist (ra) isoforms in medium supernatants of peripheral blood mononuclear cells and intestinal epithelial cells (IECs). Cytosol of macrophages which is known to contain intracellular IL-1ra (18 kDa; icIL-1 ra) and medium supernatants of macrophages known to contain secretory IL-1 ra (22-25 kDa in its glycosylated form; sIL-ra) as well as recombinant human IL-1 ra (non-glycosylated, $17 \mathrm{kDa}$; rh-IL1-ra) were used as positive controls. In the precipitated protein fraction of medium supernatants of primary IECs, only the 22-25 kDa sIL-1 ra from could be detected after 24 hours of culture. Similar results were obtained for three different IEC cultures. TNF, tumour necrosis factor. inflamed IBD mucosa (Crohn's disease, $\mathrm{p}=0.0003$; ulcerative colitis, $\mathrm{p}=0.0016$ ) but also more than inflamed mucosa from control patients (fig 3C).

Compared with IECs, LPMNCs isolated from the same patients secreted significantly more IL-8 $(p<0.0001)$ (fig 3D). In control patients, there was no increase in IL-8 secretion in LPMNCs during inflammation. In LPMNCs from non-inflamed mucosa from patients with Crohn's disease, the amount of IL-8 secretion was in the same range as for control patients. In inflammation, IL-8 secretion increased but only to 491 (75) pg/10 $10^{5}$ cells, which is in the same range as shown for the IECs. The secretion of IL-8 by LPMNCs in ulcerative colitis even in non-inflamed mucosa was much higher (3522 (365) pg/10 cells); in inflamed mucosa it increased to 4154 (488) $\mathrm{pg} / 10^{5}$ cells (fig $3 \mathrm{D}$ ).

\section{$I L-1 \beta$ and $I L-6$}

IL-1 $\beta$ was not detectable in supernatants from IECs from all patient subgroups. Also IL-6 was only present in very low amounts and was probably due to contamination with LPMNCs, supporting the results obtained from northern blot analysis and freshly isolated IECs (data not shown).

CYTOKINE SECRETION OF ILEAL AND COLONIC IECS To investigate further whether there is a difference in cytokine secretion between ileal and colonic IECs, we cultured IECs isolated from both locations for 24 hours and determined cytokine release into the medium. The values in controls, non-inflamed Crohn's disease, and inflamed Crohn's disease were not different for IL-1ra (fig 4A) and IL-8 (fig 4B), except for IL-8 secretion from inflamed Crohn's disease ileal cells. However, this difference may be due to severe terminal ileitis in these patients.

IL-1ra FORMS SECRETED BY IECs

As there are two intracellular and one secreted form of IL-ra which are supposed to have different functions, we performed western blots to determine which isoform was found in the medium of primary human IECs after 24 hours of culture. Only the glycosylated 22-25 $\mathrm{kDa}$ secretory form of IL-1 ra could be detected in the medium of IECs (fig 5). Similarly, in the medium of cultured peripheral blood mononuclear cells, the $22-25 \mathrm{kDa}$ form was predominant, but the smaller intracellular IL-1 ra form could also be detected (fig 5). Stimulation of the cells with $10 \mathrm{ng} / \mathrm{ml} \mathrm{TNF}$ for 24 hours did not change these findings.

\section{Discussion}

In this study we show that IECs from normal mucosa produce no detectable amounts of IL- $1 \beta$ or IL- 6 , whereas LPMNCs do. IECs produce and secrete very low amounts of IL-8, but in non-inflamed mucosa, much more was generated by the corresponding LPMNCs. However, IL-8 production in IECs can be induced upon stimulation. IECs secrete more IL-1ra than do the corresponding LPMNCs. 
Therefore the pattern of cytokine secretion by IECs is a more anti-inflammatory one than that of LPMNCs, which secrete all the cytokines investigated.

Several lines of evidence indicate that the data are reliable. No mRNA for IL-1 or IL-6 was seen in the IEC preparation, indicating that potential contamination with macrophages (less than $4 \%$ in FACS analysis) was not relevant to the analysis. In addition, the IEC preparation did not contain any relevant amounts of fibroblasts, as, in another series of experiments, we showed that fibroblasts secrete IL-6 even under unstimulated conditions.

Elevated levels of IL-1 ra had been previously reported in human IBD. ${ }^{29}{ }^{32}{ }^{33}$ In addition, it had been shown that IECs contribute to the IL-1ra pool of the intestinal mucosa. ${ }^{32}$ The role of IL-1ra in intestinal inflammation has been shown in animal models. Increased IL-1 ra levels have been reported in peptidoglycanpolysaccharide induced colitis in rats. ${ }^{34}$ Treatment with recombinant IL-1ra attenuated the mucosal inflammation in this model. ${ }^{34}$ Vice versa, inhibition of endogenous IL-1 ra with a neutralising antibody exacerbated formalin induced complex colitis in rabbits. ${ }^{35}$ IL-1 ra knock out mice show an increased susceptibility to endotoxaemia. ${ }^{36}$ Dextran sodium sulphate induced colitis was followed by a large increase in mortality compared with normal IL-1ra expressing mice. ${ }^{37}$ These data indicate an important role for IL-1 ra in the balance of the mucosal immune system. Our data show that IECs are the major source of IL-1ra in the mucosa. Primary IECs were able to secrete sIL-1 ra in the 24 hour culture model. This could mean that loss of epithelial cells from certain areas of the mucosa could be followed by localised imbalance of proinflammatory and anti-inflammatory cytokines. Previous studies from our laboratory have shown that the imbalance of the mucosal immune system is not specific to IBD, but can also be found in other types of colonic inflammation. ${ }^{29}$ The degree of inflammation was the most important factor for the production of IL- $1 \alpha$, IL- $1 \beta$, and IL- 1 ra.

The difference between IL-1ra secretion from IECs and LPMNCs was greatest in patients with Crohn's disease with or without inflammation (fig 3B). It is noteworthy that the ratio between IL-1ra and IL-1 was also lowest in inflamed mucosa in Crohn's disease in our previous investigations ${ }^{29}$ and that the IL-1ra levels in homogenates of mucosal biopsy specimens were lower in Crohn's disease than in ulcerative colitis. ${ }^{29}$ Impaired induction or even downregulation of IL-ra secretion in LPMNCs could worsen the imbalance of the mucosal immune system in Crohn's disease.

There have been conflicting data on the production of IL- 8 by IECs. Whereas IL- 8 secretion could be clearly induced in colonic epithelial cell lines HT- 29 and Caco- 2 by IL-1 $\beta$ and $\mathrm{TNF}^{22}$, in situ hybridisation showed IL-8 mRNA only in the lamina propria of inflamed mucosa. ${ }^{30}$ Under our in vitro conditions, LPMNCs produced significantly more IL- 8 than IECs. Only very low amounts of IL- 8 mRNA were present in cells isolated from normal mucosa. However, IL-8 secretion could be measured after 24 hours of culture of cells isolated from normal mucosa. We assume that the isolation procedure for IECs may represent an 'injury to the epithelium' inducing IL-8 production in vitro.

Our data showing lack of IL- $1 \beta$ production by IECs are in accordance with in vivo findings, as IL- $1 \beta$ protein and mRNA have been found in LPMNCs but not in IECs by most authors. ${ }^{27}{ }^{38-40}$ Only in rabbit immune complex colitis ${ }^{41}$ and acute acetic acid induced colitis in rats $^{42}$ was IL-1 production found in enterocytes.

In vivo results on IL- 6 production by IECs are contradictory, as mentioned in the introduction. Our study clearly shows that, in humans, IL-6 is not produced by epithelial cells. In earlier investigations, contamination of the cultures with fibroblasts or macrophages may have influenced the results. Interestingly, even without inflammatory stimuli, macrophages express high levels of IL-6 mRNA, especially when compared with IL-1 $\beta$ or IL-8. The physiological role of this basal IL- 6 secretion remains to be elucidated.

There is no major difference in the cytokine response between epithelial cells from ileal and colonic mucosa. The response of IECs during inflammation is not dependent on the type and whether acute or chronic mucosal inflammation is present. Therefore our study does not provide any evidence for any special "behaviour" of IECs during IBD.

The present study clarifies some important points about the function of IECs which have been controversial in the past. Human IECs do not produce IL- $1 \beta$ and do not therefore have any direct proinflammatory potential, as suggested by studies with the IEC- 6 cell line. This is very important for the interpretation of data derived from murine colitis models, as murine epithelial cells may show different behaviour from human IECs. Human IECs do not produce IL- 6 in an acute phase response. This again indicates that studies with transformed cell lines have to be interpreted very carefully. Human IECs are able to produce and secrete IL-8 upon stimulation. This may be important for the recruitment of granulocytes during acute or chronic mucosal inflammation. Human IECs are the major source of IL-1ra in the human mucosa. Upon inflammation they respond with upregulation of IL-1 ra production.

In conclusion, expression and secretion of cytokines by IECs from normal mucosa displayed a more anti-inflammatory pattern than LPMNCs. In inflamed mucosa, secretion of the anti-inflammatory secretory IL-1 ra by IECs is increased but may be antagonised by production of proinflammatory cytokines, such as IL- 8 by the IECs themselves or IL- 1 by LPMNCs.

This work was supported by the Deutsche Forschungsgemeinschaft (An 168/3-1). Parts of this manuscript were presented at the Digestive Disease Week 1997, Washington and at the Digestive Disease Week 1998, New Orleans.

1 Selby WS, Poulter LW, Hobbs S, et al. Expression of HLA-DR antigens by colonic epithelium in inflammatory bowel disease. Clin Exp Immunol 1981;44:453-8. 
2 Bland PW, Warren LG. Antigen presentation by epithelial cells of the rat small intestine. I. Kinetics, antigen specificity and blocking by anti-la antisera. Immenogy 1986 ;

Bland PW, Warren LG. Antigen presentation by epithelial cells of the rat small intestine. II. Selective p

suppressor T-cells. Immunology 1986;58:9-14.
Mayer L, Shlien R. Evidence for the function of Ia molecules on gut epithelial cells in man. $f$ Exp Med 1987; 166:1472-83

5 Mayer L, Eisenhardt D, Salomon P, et al. Expression of class II molecules on intestinal epithelial cells in humans. Differences between normal and inflammatory bowel disease. Gastroenterology 1991;100:3-12.

6 Kaiserlian D, Rigal D, Abello J, et al. Expression, function and regulation of the intercellular adhesion molecule-1 (ICAM-1) on human intestinal epithelial cell lines. Eur $\mathcal{F}$ Immunol 1991;21:2415-21.

7 Kvale D, Krajci P, Braendtzag P. Expression and regulation of adhesion molecules ICAM-1 (CD54) and LFA-3 (CD58) in human intestinal epithelial cell lines. Scand $\mathcal{F}$ Immunol 1992;35:669-76.

8 Dippold W, Wittig B, Schwaeble W, et al. Expression of intercellular adhesion molecule 1 (ICAM-1, CD 54) in colonic epithelial cells. Gut. 1993;34:1593-7.

9 McKay DM, Perdue MH. Intestinal epithelial function: the case for immunophysiological regulation. Cells and mediators (1). Dig Dis Sci 1993;38:1377-87.

10 Panja A, Barone A, Mayer L. Stimulation of lamina propria lymphocytes by intestinal epithelial cells: evidence for recognition of nonclassical restriction elements. $\mathcal{f}$ Exp Med 1994;179:943-50.

11 Jung HC, Eckmann L, Yang SK, et al. A distinct array of proinflammatory cytokines is expressed in human colon epithelial cells in response to bacterial invasion. $f$ Clin Invest 1995;95:55-65.

12 Eckmann L, Reed SL, Smith JR, et al. Entamoeba histolytica trophozoites induce an inflammatory cytokine response by cultured human cells through the paracrine action of cytocultured human cells through the paracrine action of cytolytically re.

13 Gibson P, Rosella O. Interleukin 8 secretion by colonic crypt cells in vitro: response to injury suppressed by butyrate and enhanced in inflammatory bowel disease. Gut 1995;37 $536-43$.

14 Mahida YR, Makh S, Hysde S, et al. Effect of Clostridium difficile toxin A on human intestinal epithelial cells: induction of interleukin 8 production and apoptosis after cell detachment. Gut 1996;38:337-47.

15 Spriggs DR, Imamura K, Rodriguez C, et al. Tumor necrosis factor expression in humen epithelial tumor cell lines. $\mathscr{f}$ Clin Invest 1988;81:455-60.

16 Shirota K, LeDuy L, Yuan SY, et al. Interleukin-6 and its receptors are expressed in human intestinal epithelial cells. Virchows Arch B Cell Pathol 1990;58:303-8.

17 Hedges S, Svensson M, Svanborg C. Interleukin-6 response of epithelial cell lines to bacterial stimulation in vitro. Infect Immun 1992;60:1295-301.

18 Eckmann L, Jung HC, Schürer-Maly C, et al. Differential cytokine expression by human intestinal epithelial cell
lines: regulated expression of interleukin-8. Gastroenterology lines: regulated exp

19 Dinarello CA. The biological properties of interleukin-1. Eur Cytokine Netw 1994;5:517-31.

20 Kishimoto T, Akira S, Narazaki M, et al. Interleukin-6 family of cytokines and gp 130. Blood 1995;86:1243-54.

21 Baggiolini M, Dewald B, Moser B. Interleukin-8 and related chemotactic cytokines: CXC and CC chemokines. $A d v$ Immunol 1994;55:97-179.

22 Gross V, Andus T, Daig R, et al. Regulation of interleukin-8 production in a human colon epithelial cell line (HT-29). Gastroenterology 1995;108:653-61.

23 Schuerer-Maly CC, Eckmann L, Kagnoff MF, et al. Colonic epithelial cell lines as a source of interleukin-8: stimulation by inflammatory cytokines and bacterial lipopolysaccharide. Immunology 1994;81:85-91.
24 McGee DW, Beagley KW, Aicher WK, et al. Transforming growth factor-beta enhances interleukin-6 secretion by intestinal epithelial cells. Immunology 1992;77:7-12.

25 Parikh AA, Salzmann AL, Kane CD, et al. IL-6 production in human intestinal epithelial cells following stimulation with IL-1 beta is associated with activation of the transcription factor NF-kappa B. 7 Surg Res 1997;69:139-44.

26 Weinstein DL, O'Neill BL, Metcalf ES. Salmonella typhi stimulation of human intestinal epithelial cells induced secretion of epithelial-cell derived interleukin-6. Infect Immun 1997;65:395-404.

27 Woywodt A, Neustock P, Kruse A, et al. Cytokine expression in intestinal mucosal biopsies. In situ hybridisation of the mRNA for interleukin-1 beta, interleukin-6 and tumor necrosis factor-alpha in inflammatory bowel disease. Eur Cytokine Netw 1994;5:387-95.

28 Bao S, Goldstone S, Husband AJ. Localization of IFNgamma and IL- 6 mRNA in the murine intestine by in situ hybridization. Immunology 1993;80:666-70.

29 Andus T, Daig R, Vogl D, et al. Imbalance of the interleukin 1 system in colonic mucosa: association with intestinal inflammation and interleukin 1 receptor antagonist genotype 2. Gut 1997;41:651-7.

30 Casini Raggi V, Kam L, Chong YJ, et al. Mucosal imbalance of IL-1 and IL-1 receptor antagonist in inflammatory bowel disease. A novel mechanisms of chronic intestinal inflammation. F Immunol 1995;154:2434-40.

31 Daig R, Andus T, Aschenbrenner E, et al. Increased interleukin 8 expression in the colon mucosa of patients with inflammatory bowel disease. Gut 1996;38:216-22.

32 Rogler G, Daig R, Aschenbrenner E, et al. Establishment of long-term primary cultures of human small and large intestinal epithelial cells. Lab Invest 1998;78:889-90.

33 Isaacs KL, Sartor RB, Haskill S. Cytokine messenger RNA profiles in inflammatory bowel disease mucosa detected by polymerase chain reaction amplification. Gastroenterology 1992;103:1587-95.

34 McCall RD, Haskill S, Zimmermann EM, et al. Tissue interleukin-1 and interleukin-1 receptor antagonist expression in enterocolitis in resistant and susceptible rats. Gastroenterology 1994;106:960-72.

35 Ferretti M, Casini Raggi V, Pizarro TT, et al. Neutralization of endogenous IL-1 receptor antagonist exacerbates and prolongs inflammation in rabbit immune colitis. 7 Clin Invest 1994;94:449-53.

36 Hirsch E, Irikura VM, Paul SM, et al. Functions of interleukin 1 receptor antagonist in gene knockout and overproducing mice. Proc Natl Acad Sci USA 1996;93: $11008-13$.

37 Melani L, Hirsch E, Guanzon M, et al. Deletion of the IL-1 receptor antagonist gene increases susceptibility to experimental colitis in mice [abstract]. Gastroenterology 1997;112: A1040.

38 Mahida YR, Wu K, Jewell DP. Enhanced production of interleukin 1-beta by mononuclear cells isolated from mucosa with active ulcerative colitis of Crohn's disease. Gut 1989;30:835-8.

39 Youngman KR, Simon PL, West GA, et al. Localization of intestinal interleukin 1 activity and protein and gene expression to lamina propria cells. Gastroenterology 1993; 104:749-58.

40 Panja A, Siden E, Mayer L. Synthesis and regulation of accessory/proinflammatory cytokines by intestinal epithelial cells. Clin Exp Immunol 1995;100:298-305.

41 Cominelli F, Nast CC, Duchini A, et al. Recombinant interleukin-1 receptor antagonist blocks the proinflammatory activity of endogenous interleukin-1 in rabbit immune colitis. Gastroenterology 1992;103:65-71.

42 Radema SA, van Deventer SJH, Cerami A. Interleukin 1 beta is expressed predominantly by enterocytes in experimental colitis. Gastroenterology 1991;100:1180-6. 